\title{
A GENERALIZATION OF A THEOREM OF FENCHEL
}

\author{
OLOF HANNER AND HANS RÅDSTRÖM
}

1. The following lemma in the theory of convex sets is well known (see $[1, \S 10]) .^{1}$

Lemma. Let $M$ be a given set of points in a euclidean $n$-dimensional space and let $p$ be a point of the convex hull of $M$. It is then possible to find $n+1$ points $p_{0}, p_{1}, \cdots, p_{n}$ (not necessarily different) of $M$ and $n+1$ real numbers $\lambda_{0}, \lambda_{1}, \cdots, \lambda_{n}$ so that $\lambda_{i} \geqq 0, \sum_{i=0}^{n} \lambda_{i}=1$, and $p=\sum_{i=0}^{n} \lambda_{i} p_{i}$.

In general, the number $n+1$ in this lemma cannot be replaced by any smaller number. The purpose of this note is to prove a theorem from which we deduce a necessary and sufficient condition that a given point $p$ lie in the convex hull of a set of only $n$ points belonging to a given compact set $M$.

2. We introduce the following notations and definitions.

We denote by $M$ a given set in an $n$-dimensional euclidean space $E^{n}$. We denote the convex hull of any set $A \subset E^{n}$ by $H A$.

Definition. A point $p \in E^{n}$ has the $k$-point property ( $k$-p.p.) with respect to $M$ if there exist $k$ (or fewer) points $q_{1}, q_{2}, \cdots, q_{k} \in M$ such that $p \in H\left\{q_{i}\right\}$.

If $p$ has the $k$-p.p. with respect to $M$ then $p \in H M$.

Definition. If each point $p \in H M$ has the $k$-p.p. with respect to $M$, then $M$ is said to have the $k$-p.p.

In this terminology the lemma in $\$ 1$ says that any set $M \subset E^{n}$ has the $(n+1)$-p.p. Fenchel has shown ${ }^{2}[2$, p. 241 , Satz A] that if $M$ is connected and compact, then $M$ has the $n$-p.p. As was pointed out by Bunt [3, p. 23, Stelling 15] the compactness condition of this theorem is superfluous and "connected" can be replaced by "having at most $n$ components." Bunt's argument is essentially the following.

Suppose that $p \in H M$ does not have the $n$-p.p. with respect to $M$. Then $p$ is an interior point of an $n$-simplex with the $n+1$ vertices in $M$. Reflect this simplex in the point $p$ and erect with $p$ as vertex the open convex cone on each of the faces of the reflected simplex. It is easy to verify that each such cone contains a point of $M$, namely a vertex of the original simplex. The $n+1$ cones are disjoint and the

Received by the editors June 25,1950 .

${ }^{1}$ Numbers in brackets refer to the references at the end of the paper.

2 In the hypothesis of his theorem, the words "und zusammenhängende" should obviously be added. 
union of their closures is the whole space $E^{n}$. Furthermore there can be no point of $M$ on the boundary of a cone. For if this were the case this point together with $n-1$ of the vertices of the original simplex would determine an $(n-1)$-simplex containing $p$, in contradiction to the fact that $p$ does not have the $n$-p.p. Therefore the boundaries of the cones divide $M$ into $n+1$ nonempty parts which are not mutually connected.

Thus Bunt showed that if there exists a point $p \in H M$ not having the $n$-p.p. with respect to $M$ (that is to say if $M$ does not have the $n$-p.p.), then $M$ can be divided into $n+1$ parts by the boundaries of convex cones. Fenchel, however, assuming $M$ compact, proved the existence of a plane dividing $M$. The proof of the following theorem is an adaptation of Fenchel's argument.

3. TheOREM. Let $M$ be a compact set in the $n$-dimensional euclidean space $E^{n}$ and $A$ a closed subset of $M$. Suppose that $p \in E^{n}$ is a point which does not have the $n$-p.p. with respect to $M$ and which lies outside of $H A$. Then there is a plane through p meeting neither $M$ nor $H A$.

Proof. We shall give the proof in an intuitive formulation. It is easy to arithmetize it but this would require more space and obscure the main ideas. Following Fenchel we first observe that it is enough to prove the theorem for the case in which $M$ is a finite union of closed spheres and $A$ has interior points. Suppose namely the theorem proved in this case. Then we obtain the general case in the following way.

Let $M$ and $A$ be the given sets and let $M^{n}$ denote the set of all points of $E^{n}$ having the $n$-p.p. with respect to $M$. Then $M^{n}$ is compact, and $p$ is not contained in $M^{n}$. Choose a positive number $\epsilon$ smaller than the distance from $p$ to $M^{n}$. Using the compactness of $M$ we now construct $M_{1} \supset M$ as the union of a finite number of closed $\epsilon$-spheres with centers in $M$.

Since $A$ is compact and hence $p$ has a positive distance from $H A$, we can find a closed set $A_{1}$ with interior points such that $A \subset A_{1} \subset M_{1}$ and such that $p \notin H A_{1}$.

From the way $M_{1}$ and $A_{1}$ are determined it is easy to verify that $p$ still satisfies the conditions of the theorem with respect to $M_{1}$ and $A_{1}$. Therefore, if we suppose the theorem proved for $M_{1}$ and $A_{1}$, it immediately follows for the subsets $M$ and $A$.

It remains to prove the theorem when $M$ is a finite union of spheres and $A$ contains interior points. Therefore suppose that $M$ and $A$ are such sets.

Let us consider the set $\Sigma$ of all planes through $p$ which are either 
bounding planes or supporting planes of $H A$. It will be sufficient to prove that there is at least one of the planes in $\Sigma$ which does not intersect $M$. For if such a plane had a point in common with $H A$, it would be a supporting plane to $H A$ and hence also meet $A$, a contradiction since $A \subset M$.

We topologize $\Sigma$ in the natural way (the topology of unit direction vectors). This makes $\Sigma$ compact. Let $S$ be an arbitrary plane in $\Sigma$. Denote by $S^{+}$the closed half-space determined by $S$ and containing $A$ (since $A$ has interior points this half-space is uniquely determined). Let $S^{-}$be the other closed half-space determined by $S$.

For each $S \in \Sigma$ let $E(S)$ be the volume of $S^{+} \cap M$. Then $E(S)$ is a continuous function defined on a compact space. Hence there exists a plane $S_{1}$ such that

$$
E\left(S_{1}\right)=\sup _{S \in \Sigma} E(S) .
$$

We now show that $S_{1}$ or some $S$ near it has a vacuous intersection with $M$.

Suppose $S_{1} \cap M=N \neq 0$. Since $p$ does not have the $n$-p.p. with respect to $M$, and since $N$ is in an $(n-1)$-dimensional plane and is a subset of $M$, the lemma yields $p \notin H N$. Hence there is in $S_{1}$ an $(n-2)$ dimensional plane $T$ which does not meet $H N$ (and hence not $M$ ) and which divides $S_{1}$ into two closed half-planes $U \supset N$ and $V$.

Let us consider $(n-1)$-dimensional planes through $T$ and close to $S_{1}$, which we can think of as being obtained by turning $S_{1}$ through a small angle around $T$. We consider separately the effect of turning on $V \cap M$ and $U \cap M$.

( $\alpha$ ) Since $M$ is compact, a sufficiently small turning of $S_{1}$ in either direction will produce a plane with the property $V \cap M=0$.

$(\beta)$ A sufficiently small turning of $S_{1}$ in the direction that moves $U$ into $S_{1}^{-}$will produce a plane $S$ for which $U \cap M=0$. For otherwise there would be points of $M$ between $S_{1}$ and $S$ on the same side as $U$ (but according to $(\alpha)$ none on the same side as $V$ ), which, since $M$ is a finite union of solid spheres, would mean $E(S)>E\left(S_{1}\right)$.

This contradicts (1) and thus completes the proof.

REMARK. If $A=M$ our theorem reduces to the well known theorem giving the existence of bounding planes. We have, however, used this theorem, since we needed the fact that $\Sigma$ is nonvacuous.

4. Definition. A set $M$ is called convexly connected if there is no $(n-1)$-plane $\Pi$ such that $\Pi \cap M=0$ and $M$ contains points in both the open half-spaces determined by $\Pi$.

EXAmples. Take $n=2$. (1) $M$ consists of a number of concentric 
circles. (2) $M$ consists of a hyperbola and one of its asymptotes.

The union of convexly connected sets having a point in common is convexly connected. Hence we can make the following definition.

Definition. Let $M$ be any set in $E^{n}$ and $p \in M$. The union of all convexly connected subsets of $M$ containing $p$ will be denoted by $M_{p}$ and called the convexly connected component of $p$.

If $q \in M_{p}$, then $M_{p}=M_{q}$. Hence there is a unique decomposition of $M$ into convexly connected components.

CoROllaRy 1. If $M \subset E^{n}$ is compact and has at most $n$ convexly connected components (in particular if $M$ is convexly connected) then $M$ has the n-p.p.

Corollary 2. Suppose that $M \subset E^{n}$ is a compact set and let $p$ be an arbitrary point in $E^{n}$. If $p$ does not have the $n$-p.p. with respect to $M$, then for every set $q_{0}, q_{1}, \cdots, q_{n} \in M$ such that $p \in H\left\{q_{0}, \cdots, q_{n}\right\}$ there is an $(n-1)$-plane through $p$ not intersecting $M$ and separating $q_{0}$ from $q_{1}, \cdots, q_{n}$.

Proofs. We first prove Corollary 2. Let the set $A$ of the theorem be $q_{1}, \cdots, q_{n}$. The theorem gives a plane which obviously separates $q_{0}$ from the rest of the points.

Now suppose Corollary 1 false. Then there is a point $p \in H M$, which does not have the $n$-p.p. However by the lemma there exist $q_{0}, q_{1}, \cdots, q_{n} \in M$ with $p \in H\left\{q_{0}, q_{1}, \cdots, q_{n}\right\}$. Thus by Corollary 2 any $q_{i}$ can be separated from the other $n$ points $q$ by a plane which does not meet $M$, so that each $q_{i}$ has to belong to a convexly connected component distinct from that of any other $q$. Thus $M$ has at least $n+1$ convexly connected components.

REMARK 1 . Take $n=2$. Let $(r, \phi)$ be polar coordinates. Take

$$
\begin{aligned}
& A_{i}=\left\{(r, \phi) \mid 0<r \leqq 1, \frac{2 \pi}{3} i \leqq \phi<\frac{2 \pi}{3} i+\frac{\pi}{3}\right\} \quad(i=0,1,2), \\
& M=A_{0} \cup A_{1} \cup A_{2} .
\end{aligned}
$$

This set is convexly connected (but not connected). The origin does not have the 2-p.p. This example shows that Corollary 1 (and therefore Corollary 2 and the theorem) may be false for noncompact sets. $M$ is bounded but not closed. It is also possible to give an example of a closed but not bounded set for which Corollary 1 fails to hold.

REMARK 2. That the converse of Corollary 2 is true is obvious. Thus the condition stated is both necessary and sufficient for $p$ to have the $n$-p.p. with respect to $M$. 


\section{REFERENCES}

1. E. Steinitz, J. Reine Angew. Math. vol. 143 (1913) pp. 128-275.

2. W. Fenchel, Über Krilmmung und Windung geschlossener Raumkurven, Math. Ann. vol. 101 (1929) pp. 238-252.

3. L. N. H. Bunt, Bijdrage tot de theorie der convexe puntverzamelingen, Amsterdam, 1934.

UNIVERSITY OF STOCKHOLM

\section{ON APPELL POLYNOMIALS}

R. S. VARMA

1. Sheffer [1] ${ }^{1}$ and Thorne [2] have, in their papers, given Stieltjes integral characterization to Appell sets of polynomials, defined by

$$
P_{n}^{\prime}(x)=P_{n-1}(x) .
$$

The generating function $A(t)$ of an Appell set $\left[P_{n}(x)\right]$ is given by

$$
e^{t x} A(t)=\sum_{n=0}^{\infty} t^{n} P_{n}(x) .
$$

According to Sheffer, a polynomial $P_{n}(x)$ is an Appell set if and only if there is a function $\beta(x)$ of bounded variation on $(0, \infty)$ with the following properties:

(i) The moment constants $b_{n}=\int_{0}^{\infty} x^{n} d \beta(x)$ all exist.

(ii) $b_{0} \neq 0$.

(iii) For $n=0,1,2, \cdots$,

$$
P_{n}(x)=\int_{0}^{\infty} \frac{(x+t)^{n}}{n !} d \beta(t) .
$$

2. More generally (Sheffer, p. 741) the function $(x+t)^{n} / n$ ! can be replaced by $Q_{n}(x+t)$ where $\left\{Q_{n}(x)\right\}$ is any Appell set. Now constants $\left\{q_{k}\right\}$ exist such that

$$
Q_{n}(x+t)=q_{0} \frac{(x+t)^{n}}{n !}+q_{1} \frac{(x+t)^{n-1}}{(n-1) !}+\cdots+q_{n} .
$$

This suggests the following generalization: Let $\left\{\delta_{n}(t)\right\}$ be any sequence of functions for which the integrals

Received by the editors May 28, 1949 and, in revised form, July 14, 1950.

${ }^{1}$ Numbers in brackets refer to the references cited at the end of the paper. 